\title{
Effect of superoxide dismutase supplementation on sperm DNA fragmentation
}

\author{
Luciano Negri $^{1}$, Renzo Benaglia ${ }^{1}$, Emanuela Monti ${ }^{1}$, Emanuela Morenghi ${ }^{2}$, \\ Alessandro Pizzocaro ${ }^{3}$, Paolo E. Levi Setti ${ }^{1}$ \\ ${ }^{1}$ Humanitas Research Hospital, Department of Gynecology, Division of Gynecology and Reproductive Medicine, \\ Humanitas Fertility Center, Rozzano, Milan, Italy; \\ ${ }^{2}$ Biostatistics Unit, Humanitas Research Hospital, Rozzano, Milan, Italy; \\ ${ }^{3}$ Endocrinology Unit, IRCCS, Humanitas Research Hospital, Rozzano, Milan, Italy.
}

\begin{abstract}
Summary Background: antioxidants supplementation improves sperm quality, but few trials have analyzed the effects on sperm DNA fragmentation (SDF).

This study compares the effectiveness of SOD-based antioxidant supplementation plus hydroxytyrosol and carnosol in reducing SDF with other antioxidants without SOD, hydroxytyrosol, and carnosol.

Materials and methods: men with high SDF at baseline were selected in our clinical database. The patients taken into account had a 2-month control. SDF was measured by Sperm Chromatin Dispersion test (SCD). Untreated men were used as a control group. The remaining subjects received some oral antioxidant supplements (12 different combinations of both hydrophilic and lipophilic antioxidants), with some of them receiving nutritional support with a SOD-based antioxidant supplementation plus hydroxytyrosol and carnosol.

Results: 118 men were selected for a retrospective study. Mean age $39.3 \pm 5.4$ years. Fifteen had no treatment, 55 were treated with a SOD-based antioxidant supplementation plus hydroxytyrosol and carnosol, and 48 took some antioxidant supplements for 2 months. Clinically, variations of at least $10 \%$ in baseline values of classic semen parameters and sperm DNA fragmentation were taken into consideration. Classic seminal parameters did not vary significantly in the three groups, with the exception of viability $(p=0.001)$. We assessed which of the active substances (no. 19) in different formulations were associated with variations in SDF. In the multivariable analysis of the 7 active substances that passed the univariable analysis, only the SOD molecule appeared to be linked to an improvement in SDF $(<0.0001)$. In detail, only one patient in the control group showed a spontaneous improvement in SDF (6\%), compared to 16/48 (33\%) of those taking various oral antioxidant supplements, and $31 / 55$ (56\%) of those taking a SOD-based antioxidant supplementation plus hydroxytyrosol and carnosol.

Conclusions: SOD-based antioxidant supplementation plus hydroxytyrosol and carnosol seems to provide a better chance of improving sperm DNA integrity than other classical antioxidant molecules.
\end{abstract}

KEY WORDS: Sperm DNA fragmentation: Male infertility; Superoxide dismutase; Hydroxytyrosol; Carnosol.

Submitted 19 July 2017; Accepted 3 August 2017

\section{INTRODUCTION}

Sperm DNA fragmentation (SDF) is an important factor in the etiology of male infertility. SDF negatively correlates with pregnancy in natural cycles (1), intrauterine inseminations (2), and in-vitro procedures (3). SDF is also associated with recurrent miscarriage (4), both during in-vivo and in-vitro procedures. It is well known that SDF may be present in men with both normal and abnormal semen analysis (5) and that infertile men have higher proportions of sperm with DNA damage compared to fertile men (6). Cohen-Bacrie et al. (2009) (7) found elevated levels of sperm DNA damage in over $60 \%$ of men attending fertility clinics, with $30 \%$ being severe. Because conventional semen analysis is a poor predictor of sperm DNA damage (8), SDF assays have been suggested in selected cases of infertility, e.g. unexplained infertility, recurrent miscarriages, and asthenoteratozoospermia.

Abortive apoptosis, infection, defective spermatogenesis, and oxidative stress (OS) are thought to be causes of SDF (9), with the latter being the most common cause (10). Oxidative stress occurs when reactive oxygen species (ROS) overcome the semen's natural antioxidant defenses. In physiological conditions, oxidative stress is suitably balanced by the action of endogenous enzymatic antioxidants, including superoxide dismutase (SOD), catalase, and glutathione peroxidase/reductase, as well as non-enzymatic antioxidants such as ascorbate, urate, vitamin E, pyruvate, glutathione, albumin, vitamin A, ubiquinol, taurine, and hypotaurine (11). These endogenous antioxidants scavenge both intracellular and extracellular superoxide radicals, preventing the lipid peroxidation of plasma membranes (12). Pathological stressors that generate endogenous ROS include infections, varicocele, aging, cancer, drugs, cigarette smoking, obesity, pharmaceutics, industrial chemicals, radio-frequency electromagnetic radiation and, lastly, abnormal spermatozoa $(13,14)$. All these stressors decrease sperm motility and viability, while stimulating DNA base adduct formation and, ultimately, DNA fragmentation.

Physiologically, homeostasis between free radicals and antioxidant substances is guaranteed by very complex systems. The most efficient seems to be the system mediated by the Nrf2 (Nuclear factor [erythroid-derived 2]-like 2 transcription factor) pathway. The latter regulates a wide variety of antioxidant cytoprotective enzymes through a promotion sequence known as ARE (antioxidant response element) (15) 
At present, several tests have been developed to evaluate sperm DNA fragmentation. These include the Sperm Chromatin Dispersion test (SCD) (16), a simple and inexpensive test for basic diagnosis in clinical practice. When no clear SDF etiological factors exist, antioxidants drugs are empirically prescribed $(17,18)$. Numerous combinations of hydrophilic and lipophilic antioxidants are available in drugstores and online, e.g. vitamin C, vitamin E, folic acid, DHA, L-acetyl carnitine, L-carnitine, astaxanthin, ethyl cysteine, coenzyme Q10, zinc, and selenium. In view of the fact that recent in-vitro and in-vivo trials support the theory that Nrf2 activation strategies could effectively combat oxidative stress, the purpose of our retrospective study is to match the effectiveness of SODbased antioxidant therapy plus hydroxytyrosol and carnosol (FertiPlus ${ }^{\circledR}$ SOD) in reducing sperm DNA fragmentation with other antioxidants without SOD, hydroxytyrosol, and carnosol.

\section{MATERIALS AND METHOdS}

\section{Selection of participants and data collection}

Male partners of infertile couples referred to our Fertility Center were selected by a query in our clinical database (June 2014 - November 2016). Inclusion criteria were oligo-normozoospermia, according to the 2010 World Health Organization criteria for the Evaluation of Human Semen (5 ${ }^{\text {th }}$ Edition) (19); Sperm DNA fragmentation $>$ 15\%; no current seminal infections according to sperm culture and/or seminal leucocyte $\left(<10^{6} \mathrm{x} \mathrm{ml}\right)$; no sperm antibodies (Sperm Mar IgG; Ferti Pro, N.V., Origio, Florence, Italy); no varicocele. These patients had i) mildly or severely high levels of DNA fragmentation at baseline examination and ii) SCD control after 2 months.

The medical histories of all patients were taken into consideration and physical examinations plus ultrasonography

\section{Table 1.}

Conventional seminal parameters and clinical characteristics of patients - TPMC means total progressively motile sperm count [(volume $x$ sperm concentration $x$ progressive motility) $\left./ 10^{-8}\right]$.

\begin{tabular}{|l|c|c|c|}
\hline & At baseline & 2 Months control & $\mathbf{P}$ \\
\hline Patients & 118 & 118 & \\
\hline Infertility duration (months) & $32.9 \pm 24.8$ & & \\
\hline Male age (yrs.) & $39.4 \pm 5.4$ & & \\
\hline Female age (yrs.) & $35.1 \pm 4.3$ & & \\
\hline Male BMI & $25.7 \pm 3.0$ & & \\
\hline Total orchidometry (ml) & $28.7 \pm 8.2$ & & \\
\hline Active smoking & $27(22.88 \%)$ & & \\
\hline FSH (mu/ml) & $5.43 \pm 3.07$ & & \\
\hline Comorbidities & $35(29.66 \%)$ & & \\
\hline Medication use & $25(21.19 \%)$ & & \\
\hline Semen volume (ml) & $3.52 \pm 1.51$ & $3.43 \pm 1.55$ & 0.393 \\
\hline Total sperm count (106) & $70.4 \pm 63.2$ & $80.9 \pm 78.4$ & 0.159 \\
\hline TPMC (106) & $13.9 \pm 15.4$ & $17.2 \pm 20.0$ & 0.160 \\
\hline Progressive motility (\%) & $18.3 \pm 10.2$ & $19.3 \pm 11.4$ & 0.738 \\
\hline Normal forms (\%) & $3.49 \pm 2.09$ & $3.64 \pm 1.86$ & 0.334 \\
\hline Viability (\%) & $63.6 \pm 11.9$ & $66.6 \pm 10.4$ & 0.014 \\
\hline
\end{tabular}

of reproductive apparatus were conducted by three clinical andrologists (LN, RB and AP). Semen analyses, as well hormone profiles, were evaluated in our hospital. The presence of previous cryptorchidism, long-term medication use (e.g. selective serotonin reuptake inhibitors, tranquilizers, antihypertensives, substances for the prevention of fatty acid metabolism disorders, mesalazine), and idiopathic hypogonadism were not considered exclusion factors as they are representative of everyday real-life clinical practice. Table 1 shows the clinical characteristics of patients. This retrospective study was approved by our hospital's Institutional Ethical Committee and all patients provided written informed consent for the scientific use of their clinical data. The primary endpoint of the study was to analyze the improvement in sperm integrity (SDF reduction) after antioxidant oral supplementation. The secondary endpoints were the effects on classic semen parameters (sperm count, progressive motility, normal forms, viability) and the presence of adverse events. Pregnancy, miscarriage, and live birth rates were not considered due to the short duration of treatment (2 months).

\section{Semen processing}

Semen samples were obtained in a collection room located in the same facility as the andrology laboratory after 3-5 days of sexual abstinence. After liquefaction at $37^{\circ} \mathrm{C}$ in sterile cups, seminal volume and $\mathrm{pH}$, sperm concentration, motility, morphology, and viability were evaluated according to World Health Organization guidelines (2010) (19). We analyzed the total sperm count instead of the concentration $/ \mathrm{ml}$, as it is more representative of actual testicular function.

\section{SCD test}

The method used was Halosperm G2 ${ }^{\circledR}$ (Halotech, Madrid, Spain), in keeping with the manufacturer's protocol (http://www.halotechdna.com/wp-content/uploads/2015/ 04/IU-halosperm-G2_10det_v2.pdf). The SCD test is based on the principle that sperm with fragmented DNA fail to produce the characteristic halo of dispersed DNA loops observed in sperm with non-fragmented DNA, following acid denaturation and removal of nuclear proteins. Sperm cells with very small halos or without halos are to be considered as containing fragmented DNA.

The extent of DNA damage for each semen sample is expressed as the sperm DNA fragmentation index (SDF). In humans, a threshold of 30\% SDF is suggested as a cutoff to distinguish between a potentially fertile vs infertile semen sample, although a threshold of $18 \%$ has been suggested as predictive of a poor fertilization rate. In order to understand whether the patient achieved a clinically useful improvement in SDF, we arbitrarily selected a cut-off of $10 \%$ DNA fragmentation percentage change, calculated as:

\section{Statistics}

The data were described as number and percentage, or mean and standard deviation, as appropriate. Differences were explored with the Wilcoxon test for paired data when comparing data at baseline and 2 months control, or the Kruskal-Wallis test when comparing improvements in SDF or integrator type. The association between the percentage variation and the commercial product 
was explored with an univariable linear regression; all the products with a $\mathrm{p}$ less than 0.1 were then subjected to a multivariable linear regression.

All analyses were made with statal3 software (StataCorp LP, 4905 Lakeway Drive, College Station, TX 77845, USA). A $\mathrm{p}<0.05$ was considered as significant.

\section{RESULTS}

118 male partners of infertile couples treated from June 2014 - November 2016 were selected for a retrospective study. Mean age was $39.3 \pm 5.4$ years, infertility duration was $32.9 \pm 24.8$ months. Female age on examination was $35.1 \pm 4.3$ years. Conventional seminal parameters and clinical characteristics of patients are reported in Table 1. None of the patients reported any adverse events after oral antioxidant supplementation.

Of these men, 15 had received no medical or surgical treatment and were used as a control group.

The remaining 103 had received some oral antioxidant supplementation (12 different combinations of both hydrophilic and lipophilic antioxidants), whose composition is shown in Table 2. Fifty-five were treated with SOD-based antioxidant FertiPlus ${ }^{\circledR}$ SOD, whose formulation contains ORISOD $^{\circledR}$, Extramel $^{\circledR}, \alpha$-lipoic acid, glutathione, folic acid, zinc, and vitamins B2, B3, B6, B12. FertiPlus ${ }^{\circledR} S O D$ is a balanced combination of enzymatic and non-enzymatic antioxidants (SOD micro encapsulated [Extramel $\left.{ }^{\circledR}\right]$ ), alpha lipoic acid, glutathione (low dose), zinc, B vitamins, a micronutrient complex $\left(O R I S O D^{\circledR}\right)$ containing substances of plant origin, hydroxytyrosol, and carnosol, identified as substances able to activate the antioxidant system and detoxify intracellular endogenous NRF-2 (nuclear transcription factor-erythroid 2).

Five patients had a clinical history of juvenile orchidopexy and one had a history of previous unilateral seminoma without chemoradiotherapy; one had testicular microlithiasis, one reported a low birth weight, one had unilateral absence of the vas deferens and ipsilateral renal agenesis, and one had unilateral testicular torsion without antisperm antibodies. Another 25 patients were taking medication for anxiety and depression (n. 1), asthma (n. 6), Behçet's disease (n. 1), gastroesophageal reflux (n. 5), nasal polyposis (n. 1), hypertension (n. 3), hypothyroidism (n. 1), Crohn's disease (n. 1), juvenile diabetes (n. 1), epilepsy (n. 1), mild chronic renal insufficiency (n. 1), hypercholesterolemia (n. 1), and pudendal nerve entrapment (n. 1). One patient was using cannabis.

We then assessed which of the active substances were associated with variations in DNA fragmentation, individually considered and corrected on an individual basis for the statistically significant cases. The results are shown in Table 2. In the multivariable analysis of the seven active substances that passed the univariable analysis, only the SOD molecule appears to be linked to an improvement in SDF.

Clinically, variations of at least 10\% in baseline values of classic semen parameters and sperm DNA fragmentation were taken into consideration. Variations between the two limits were not considered clinically relevant. Table $3 \mathrm{a}$ shows the clinical and seminal parameters compared to the percentage change in SDF. No seminal parameters were associated with the variation in DNA fragmentation, except for the improvement in sperm viability $(p=0.001)$ and, to a slight extent, the improvement in progressive motility $(p=0.07)$. The distribution of comorbidities and medication use is homogeneous in the three analyzed groups. Furthermore, no differences in age, BMI, active smoking, FSH, and total testicular volume were observed in the three groups. Classic seminal parameters (total sperm count, progressive motility, and morphology) do

\begin{tabular}{|c|c|c|c|c|c|c|}
\hline Ingredients & $\begin{array}{c}\text { Commercial } \\
\text { products (n. 12) }\end{array}$ & $\begin{array}{l}\text { No. patients } \\
\text { (n. 118) }\end{array}$ & $\begin{array}{c}\text { Absent } \\
\text { (mean } \pm \text { SD) }\end{array}$ & $\begin{array}{c}\text { Present } \\
\text { (mean } \pm \text { SD) }\end{array}$ & $\underset{\text { (univariable) }}{P}$ & $\begin{array}{c}\mathrm{P} \\
\text { (multivariable) }\end{array}$ \\
\hline Vit. E & 7 & 28 & $6.96 \pm 27.83$ & $-4.66 \pm 28.47$ & 0.1189 & \\
\hline Vit. C & 6 & 33 & $7.21 \pm 26.90$ & $-3.57 \pm 30.69$ & 0.0920 & 0.2505 \\
\hline$\overline{Z i n c}$ & 6 & 82 & $-7.54 \pm 28.93$ & $9.36 \pm 26.60$ & 0.0024 & 0.3565 \\
\hline Arginine & 6 & 23 & $6.18 \pm 28.64$ & $-3.96 \pm 25.84$ & 0.1404 & \\
\hline Selenium & 5 & 16 & $5.87 \pm 28.44$ & $-6.43 \pm 25.74$ & 0.1141 & \\
\hline L-carnitine & 5 & 17 & $5.57 \pm 28.11$ & $-3.91 \pm 28.90$ & 0.2902 & \\
\hline Folic acid & 5 & 71 & $-7.36 \pm 27.23$ & $11.86 \pm 26.50$ & 0.0002 & 0.6156 \\
\hline Coenzyme Q10 & 3 & 17 & $5.3 \pm 29.63$ & $-3.70 \pm 17.11$ & 0.1341 & \\
\hline Inositol & 3 & 8 & $5.18 \pm 27.90$ & $-9.30 \pm 32.36$ & 0.2702 & \\
\hline Vit. B & 2 & 57 & $-6.54 \pm 26.55$ & $15.69 \pm 25.65$ & $<0.0001$ & 0.2226 \\
\hline Astaxanthine & 2 & 8 & $4.77 \pm 28.85$ & $-3.56 \pm 18.80$ & 0.2941 & \\
\hline$\alpha$-lipoic acid & 2 & 57 & $-6.54 \pm 26.55$ & $15.69 \pm 25.65$ & $<0.0001$ & -* \\
\hline DHA & 2 & 8 & $4.33 \pm 28.04$ & $2.47 \pm 33.80$ & 0.4799 & \\
\hline SOD & 1 & 55 & $-7.13 \pm 26.65$ & $17.18 \pm 24.46$ & $<0.0001$ & $<0.0001$ \\
\hline L-taurine & 1 & 12 & $4.81 \pm 27.78$ & $-1.14 \pm 33.47$ & 0.7997 & \\
\hline Aspartic acid & 1 & 10 & $5.02 \pm 28.95$ & $-4.60 \pm 18.90$ & 0.2308 & \\
\hline Glutathione & 1 & 60 & $-7.06 \pm 25.45$ & $15.09 \pm 26.78$ & $<0.0001$ & 0.6537 \\
\hline Tryptophan & 1 & 2 & $4.71 \pm 28.11$ & $-25.06 \pm 33.09$ & 0.1961 & \\
\hline Maca & 1 & 6 & $4.22 \pm 27.98$ & $3.93 \pm 36.88$ & 0.5648 & \\
\hline Any treatment & & 103 & $-16.15 \pm 22.39$ & $7.17 \pm 27.93$ & 0.0028 & \\
\hline
\end{tabular}

Table 2.

The table lists the substances contained in 12 commercial products.

The second column shows the number of prescriptions in the 103 patients evaluated in the study. The fourth and fifth columns report the percentage variation in DNA fragmentation for each individual active substance. Data are expressed as mean $\pm S D$. 


\begin{tabular}{|c|c|c|c|c|}
\hline & \multicolumn{3}{|c|}{ SDF variation } & \multirow[b]{2}{*}{$\mathbf{P}$} \\
\hline & $\begin{array}{c}>10 \% \\
\text { deterioration }\end{array}$ & Unchanged & $\begin{array}{c}>10 \% \\
\text { improvement }\end{array}$ & \\
\hline $\mathbf{N}$ & 36 & 34 & 48 & \\
\hline Male age (yrs.) & $38.6 \pm 4.2$ & $39.9 \pm 4.8$ & $39.6 \pm 6.5$ & 0.640 \\
\hline Female age (yrs.) & $35.2 \pm 4.3$ & $34.8 \pm 4.2$ & $35.1 \pm 4.4$ & 0.923 \\
\hline Male BMI & $25.5 \pm 2.9$ & $26.1 \pm 2.8$ & $25.5 \pm 3.2$ & 0.364 \\
\hline Total orchidometry (ml) & $30.6 \pm 7.9$ & $27.8 \pm 8.8$ & $27.8 \pm 7.9$ & 0.302 \\
\hline Infertility duration (months) & $32.8 \pm 28.6$ & $33.7 \pm 22.7$ & $32.5 \pm 23.7$ & 0.587 \\
\hline Active smoking & $6(16.67 \%)$ & $7(20.59 \%)$ & $14(29.17 \%)$ & 0.419 \\
\hline$\overline{\text { FSH }(\mathrm{mu} / \mathrm{ml})}$ & $5.20 \pm 2.90$ & $5.87 \pm 3.53$ & $5.28 \pm 2.87$ & 0.512 \\
\hline Medication use & $6(16.67 \%)$ & $6(17.65 \%)$ & $13(27.08 \%)$ & 0.471 \\
\hline Comorbidities & $8(22.22 \%)$ & $9(26.47 \%)$ & $18(37.50 \%)$ & 0.296 \\
\hline Antioxidants & & & & $<0.001$ \\
\hline None & $8(22.22 \%)$ & $6(17.65 \%)$ & $1(2.08 \%)$ & \\
\hline Other & $21(58.33 \%)$ & $11(32.35 \%)$ & $16(33.33 \%)$ & \\
\hline$\overline{S O D}$ & $7(19.44 \%)$ & $17(50.00 \%)$ & $31(64.58 \%)$ & \\
\hline Baseline semen volume (ml) & $3.47 \pm 1.40$ & $3.65 \pm 1.68$ & $3.45 \pm 1.50$ & 0.918 \\
\hline$\Delta$ Semen volume $(\%)$ & $11.5 \pm 28.7$ & $2.5 \pm 34.7$ & $-5.6 \pm 28.1$ & 0.025 \\
\hline Baseline total sperm count (106) & $80.2 \pm 79.1$ & $57.1 \pm 52.2$ & $72.4 \pm 56.3$ & 0.299 \\
\hline$\Delta$ Total sperm count $(\%)$ & $68.4 \pm 163.1$ & $18.3 \pm 77.5$ & $16.0 \pm 68.9$ & 0.307 \\
\hline Baseline TPMC (106) & $17.1 \pm 19.7$ & $10.3 \pm 9.5$ & $14.1 \pm 14.8$ & 0.4971 \\
\hline$\triangle$ TPMC $(\%)$ & $98.6 \pm 179.1$ & $27.0 \pm 124.1$ & $83.9 \pm 269.7$ & 0.204 \\
\hline Baseline progressive motility (\%) & $19.8 \pm 11.5$ & $17.0 \pm 7.7$ & $18.3 \pm 10.8$ & 0.6110 \\
\hline$\Delta$ Progressive motility (\%) & $11.8 \pm 55.0$ & $-3.4 \pm 43.4$ & $43.3 \pm 115.6$ & 0.074 \\
\hline Baseline normal forms (\%) & $3.36 \pm 2.17$ & $3.32 \pm 2.40$ & $3.71 \pm 1.82$ & 0.2039 \\
\hline$\Delta$ Normal forms (\%) & $10.2 \pm 54.0$ & $25.0 \pm 70.0$ & $54.6 \pm 161.0$ & 0.717 \\
\hline Baseline viability & $63.4 \pm 11.6$ & $65.5 \pm 8.2$ & $62.4 \pm 14.2$ & 0.8646 \\
\hline$\overline{\Delta \text { Viability (\%) }}$ & $2.9 \pm 21.9$ & $-0.4 \pm 12.5$ & $20.0 \pm 47.5$ & 0.001 \\
\hline
\end{tabular}

\begin{tabular}{|lcccc}
\hline $\mathbf{N}$ & No drugs & Other drugs & SOD & $\mathbf{P}$ \\
\hline Male age (yrs.) & 15 & 48 & 55 & \\
\hline Female age (yrs.) & $38.7 \pm 4.4$ & $38.9 \pm 5.5$ & $40.0 \pm 5.6$ & 0.454 \\
\hline Male BMI & $34.3 \pm 5.2$ & $35.1 \pm 3.5$ & $35.2 \pm 4.6$ & 0.709 \\
\hline Total orchidometry (ml) & $24.8 \pm 2.1$ & $25.6 \pm 2.7$ & $26.0 \pm 3.4$ & 0.441 \\
\hline Infertility duration (months) & $30.7 \pm 7.4$ & $29.6 \pm 8.9$ & $27.3 \pm 7.7$ & 0.421 \\
\hline Active smoking & $34.7 \pm 21.5$ & $32.3 \pm 24.8$ & $33 \pm 26.1$ & 0.754 \\
\hline FSH (mu/ml) & $1(6.67 \%)$ & $14(29.17 \%)$ & $12(21.82 \%)$ & 0.202 \\
\hline Medication use & $4.39 \pm 1.16$ & $5.72 \pm 3.59$ & $5.46 \pm 2.91$ & 0.464 \\
\hline Comorbidities & $3(20.00 \%)$ & $12(25.00 \%)$ & $10(18.18 \%)$ & 0.703 \\
\hline Baseline SDF & $3(20.00 \%)$ & $13(27.08 \%)$ & $19(34.55 \%)$ & 0.521 \\
\hline$\Delta$ SDF (\%) & $34.9 \pm 12.5$ & $37.0 \pm 9.5$ & $40.0 \pm 12.6$ & 0.3728 \\
\hline Baseline semen volume (ml) & $16.1 \pm 22.4$ & $4.3 \pm 27.5$ & $-17.2 \pm 24.5$ & $<0.001$ \\
\hline$\Delta$ Semen volume (\%) & $3.64 \pm 1.26$ & $3.60 \pm 1.55$ & $3.40 \pm 1.56$ & 0.639 \\
\hline Baseline total sperm count (106) & $8.7 \pm 27.5$ & $2.5 \pm 31.4$ & $-0.5 \pm 31.5$ & 0.596 \\
\hline$\Delta$ Total sperm count (\%) & $76.4 \pm 90.0$ & $62.4 \pm 50.8$ & $75.7 \pm 64.9$ & 0.825 \\
\hline Baseline TPMC (106) & $52.1 \pm 78.2$ & $45.5 \pm 145.4$ & $16.1 \pm 75.7$ & 0.165 \\
\hline$\Delta$ TPMC (\%) & $15.1 \pm 15.7$ & $11.8 \pm 11.7$ & $15.5 \pm 17.9$ & 0.857 \\
\hline Baseline progressive motility (\%) & $74.6 \pm 144.5$ & $63.1 \pm 155.4$ & $79.0 \pm 263.4$ & 0.644 \\
\hline$\Delta$ Progressive motility (\%) & $22.2 \pm 13.4$ & $17.7 \pm 8.5$ & $17.9 \pm 10.6$ & 0.473 \\
\hline Baseline normal forms (\%) & $11.1 \pm 57.1$ & $9.2 \pm 49.2$ & $32.2 \pm 111.7$ & 0.529 \\
\hline$\Delta$ Normal forms (\%) & $2.53 \pm 1.41$ & $3.60 \pm 2.16$ & $3.65 \pm 2.15$ & 0.229 \\
\hline Baseline viability & $45.5 \pm 98.1$ & $15.1 \pm 58.9$ & $44.9 \pm 150.3$ & 0.483 \\
\hline$\Delta$ Viability (\%) & $68.1 \pm 11.0$ & $63.6 \pm 10.1$ & $62.4 \pm 13.5$ & 0.161 \\
\hline
\end{tabular}

Table 3a.

Clinical and seminal parameters compared to the percentage change in SDF.

The $\Delta$ are calculated as (post-pre)/pre and expressed as a percentage.
Table $3 b$.

Clinical and seminal parameters in the three groups. Classic seminal parameters (total sperm count, progressive motility, and morphology) do not vary significantly in the three groups, except for viability and SDF, showing an improvement in the group receiving SOD-

The $\Delta$ are calculated as (post-pre)/pre and are expressed as a percentage. not vary significantly in the three groups, except for SDF, showing an improvement in the group receiving SOD (Table 3b). In particular the post-hoc evalution of SDF variation test power (by Cohen's d) is greater than 0.98 confirming the adeguacy of sample size. In greater detail, only one patient in the control group showed a spontaneous improvement in SDF (6\%), compared to $16 / 48$ (33\%) of those taking various oral antioxidant supple- 
ments and 31/55 (56\%) of those taking oral antioxidant supplements with SOD. Nevertheless, it should be considered that although fragmentation can also improve spontaneously in patients with risk factors for comorbidities or drug therapy, the positive impact of the integrator administration persists, succeeding in combatting the oxidative damage caused by free radicals and highly reactive oxygen species, which have been identified as the agents responsible for sperm DNA damage.

\section{Discussion}

It is believed that about 80 million people worldwide are affected by the inability to have children (20), with male factor subfertility accounting for up to $50 \%$ of these cases (21). Some $30-80 \%$ of male factor subfertility cases are believed to be due to the damaging effects of oxidative stress (21). Oral supplementation with antioxidants is thought to improve sperm quality by reducing oxidative stress (22) and these products are widely available and inexpensive when compared to other fertility treatments. This suggestion is so widely spread by the media that, currently, a high percentage of couples turning to our Fertility Center are already taking antioxidants, prescribed by gynecologists, general practitioners, or even self-prescribed.

At present, several tests have been developed to evaluate sperm DNA fragmentation, e.g. TUNEL (TdT-mediated dUTP nick-end labeling) (23), Comet Assay (24), Sperm Chromatin Structure Assay (SCSA) (25) and Sperm Chromatin Dispersion test (SCD) (16). While TUNEL and Comet Assay directly detect DNA damage (the latter also finding single and double strand breaks), SCSA and SCD measure DNA fragmentation after a mild denaturation process. TUNEL and SCSA employ flow cytometry, with little intra-technician variability. However, they are complex, time consuming, and expensive (flow cytometer). Comet Assay is not suited for rapid diagnosis and requires highly specialized personnel to analyze the results.

The SCD test is a simple and inexpensive technique, but could have higher intra-individual variation.

There are currently six meta-analyses of antioxidant treatment for male infertility available $(22,26-30)$ and all report improvements in pregnancy rate and sperm quality after therapy. Ross et al. (2010) (29) report improvement in at least one semen variable in 13 out of 17 studies analyzed. In a more recent Cochrane meta-analysis (22), comprising 48 studies, 4.179 men were analyzed; of these, 2.466 received oral antioxidant supplementation and 1.713 received no treatment. The patient population was made up of the male partners of couples who had attended a fertility clinic. Surprisingly, only two trials performed on a total of 100 patients $(64+36)$ analyzed the effects of oral antioxidant supplementation on $\operatorname{SDF}(31,32)$ and both observed a reduction in SDF when compared to placebo (mean difference: $-13.85,95 \%$ CI -17.28 to $-10.41, \mathrm{P}<$ 0.00001). One investigator used vitamin $C+$ vitamin $E$, while the other used docosahexaenoic acid (DHA). Menezo et al. (2007) (33) (not included in the meta-analysis) treated 58 men with an SDF > 15\% with oral antioxidant therapy (vitamins $\mathrm{C}$ and $\mathrm{E}$, beta carotene, zinc and selenium) for 13 weeks and reported a significant improvement in DNA fragmentation $(-19.1 \%, \mathrm{p}<0.0004)$.
Our data are not all consistent with those reported in literature, not providing a significant improvement in classic seminal parameters (total sperm count, progressive motility, and morphology). Basic semen parameters do not vary significantly in the three groups (antioxidants, FertiPlus ${ }^{\circledR}$ SOD, no medication), except as regards viability. The reasons may be related to the older age of our population $(39.4 \pm 5.4$ yrs. $)$, which reflects the later age at which couples are deciding to have children. Secondly, the selection of patients was as close as possible to everyday real-life clinical practice. Indeed, we only excluded patients with varicocele and seminal infections, as diseases associated with SDF, but susceptible to effective specific treatment (antibiotics and surgery). Patients with antisperm antibodies were excluded as in other studies, although the two available cases did not have a high degree of DNA fragmentation (data not shown). Most of the studies published to date did not enroll men with a considerable number of risk factors, such as smoking, recreational drug use, systemic diseases, longterm medication use, alcohol, oligozoospermia, high serum gonadotropins, previous orchidopexy, and anatomic abnormalities of the genital tract. While this approach permits a better appreciation of the effect of medical treatment, it also drastically reduces the number of candidates for oral antioxidant treatment. At our Fertility Center, perfectly healthy, young patients without any bad habits are really very few.

Our selection criteria could, therefore, justify unsatisfactory results in terms of classic sperm parameters. Antioxidants not containing SOD led to an improvement of at least $10 \%$ in TPMC in $43.8 \%$ patients vs $45.5 \%$ in men treated with FertiPlus ${ }^{\circledR}$ SOD (n.s.). In the same two groups sperm morphology increased by at least 10\% to $31.3 \%$ and $36.4 \%$, n.s.), respectively, while oral antioxidant supplementation proved effective in reducing sperm DNA fragmentation. As mentioned in the Results section, only one patient in the control group showed a spontaneous improvement in SDF (6\%), compared to $16 / 48$ (33\%) of those taking various oral antioxidant supplements and $31 / 55(56 \%)$ of those taking oral antioxidant supplements with SOD ( $<<0.0001)$.

From a clinical viewpoint, the possibility to reduce sperm DNA fragmentation in 56\% of otherwise untreatable infertile patients is certainly an ethically and economically sound approach. We must therefore consider that almost one quarter of our patients had untreatable diseases, requiring long-term treatment; 13 had high FSH $(7.6-21.1 \mathrm{mu} / \mathrm{ml}), 17$ had a testicular volume of less than $12 \mathrm{ml}, 10$ had class 1 obesity, and 27 were active smokers. Nevertheless, we were surprised to observe that the presence of co-morbidities, signs of testicular impairment and bad habits did not affect the chances of improving DNA fragmentation.

While oral SOD supplementation seems to work better than any other antioxidant molecules analyzed, it remains unclear why the benefit is observed in just over half of the cases treated. One could assume that 2 months intake are insufficient to fully express the therapeutic effect. Another possible explanation may be that antioxidant therapy could be ineffective if given to males whose subfertility is not caused by oxidative stress (34) and, in this respect, no 
patients underwent an objective test indicating that oxidative stress was the key factor behind their condition. Our study has a number of limitations. Firstly, it is retrospective, meaning that neither a causality hypothesis nor mechanistic models can be drawn up due to the nature of our study. Secondly, the data derive from patients entering an IVF-ICSI program, who could have different characteristics from the general male population. In addition, another limitation is the low number of subjects examined. Lastly, the seminal OS levels were not assessed. Although to be confirmed in a randomised trial this result is a new and relevant data in patient's counselling.

\section{Conclusions}

Oral SOD supplementation appears to produce a better reduction in sperm DNA fragmentation in the infertile population than other commonly used antioxidant formulations. When used in unselected infertile patients, representative of daily clinical practice, FertiPlus ${ }^{\circledR}$ SOD reduces DNA fragmentation in $56 \%$ of cases compared to $33 \%$ of cases using other antioxidant formulations. Therefore, given the absolute tolerability of the product and the affordable cost, this approach is to be considered clinically and ethically acceptable.

\section{AUTHOR CONTRIBUTIONS}

NL provided the study design concept, drafted the article and interpreted the data.

NL, RB and AP recruited the subjects, compiled the medical records, performed physical examinations and the color-Doppler ultrasound evaluations.

EM performed the SCD test and semen analyses

EM performed the statistical analyses

PELS made a substantial contribution to critically revising the article.

\section{EthICS APPROVAL AND CONSENT TO PARTICIPATE}

We declare that our study has been conducted according to the Helsinki Declaration on clinical research and to the Ethical Code on animal research set forth by WHO (WHO Chronicle 1985; 39:51) and that has been approved by IRCCS Istituto Clinico Humanitas INDEPENDENT ETHICS COMMITTEE, reference number $1 / 17$, on January 17, 2017. A written informed consent was obtained from each participant before study.

\section{References}

1. Spano M, Bonde J, Hjøllund HI, et al. Sperm chromatin damage impairs human fertility. Fertil Steril. 2000; 73:43-50.

2. Bungum M, Humaidan P, Axmon A, et al. Sperm DNA integrity assessment in prediction of assisted reproduction technology outcome. Hum Reprod. 2007; 22:174-9.

3. Osman A, Alsomait H, Seshadri S, et al. The effect of sperm DNA fragmentation on live birth rate after IVF or ICSI: a systematic review and meta-analysis. RBM Online. 2015; 30:120-7.

4. Lewis SE, Aitken RJ. DNA damage to spermatozoa has impacts on fertilization and pregnancy. Cell Tissue Res. 2005; 322:33-41.
5. Erenpreiss J, Elzanaty S, Giwercman A. Sperm DNA damage in men from infertile couples. Asian J Androl. 2008; 10:786-90.

6. Saleh RA, Agarwal A, Nelson DE, et al. Increased sperm nuclear DNA damage in normozoospermic infertile men: a prospective study. Fertil Steril. 2002; 78:313-8.

7. Cohen-Bacrie P, Belloc S, Menezo YJ, et al. Correlation between DNA damage and sperm parameters: a prospective study of 1,633 patients. Fertil Steril. 2009; 91:1801-5.

8. Virro, MR, Larson-Cook, KL, Evenson DP. Sperm chromatin structure assay (SCSA) parameters are related to fertilization, blastocyst development, and ongoing pregnancy in in vitro fertilization and intracytoplasmic sperm injection cycles. Fertil Steril. 2004; 81:1289-95.

9. Aitken RJ, Wingate JK, De Iuliis GN, et al. Analysis of lipid peroxidation in human spermatozoa using BODIPY C11. Mol Hum Reprod. 2007; 13:203-11.

10. Agarwal A, Makker K, Sharma R. Clinical Relevance of Oxidative Stress in Male Factor Infertility: An Update. Am J Reprod Immunol. 2008; 59:2-11.

11. Aitken RJ, De Iuliis GN.. On the possible origins of DNA damage in human spermatozoa. Mol Hum Reprod. 2010; 16:3-13.

12. Aitken RJ, Clarkson JS, Fishel S. Generation of reactive oxygen species, lipid peroxidation and human sperm function. Biol Reprod. 1989; 40:183-97.

13. De Iuliis GN, Newey RJ, King BV, et al. Mobile phone radiation induces reactive oxygen species production and DNA damage in human spermatozoa in vitro. PLoS One 2009; 4:e6446.

14. Wright C, Milne S, Leeson H. Sperm DNA damage caused by oxidative stress: modifiable clinical, lifestyle and nutritional factors in male infertility. RBM Online. 2014; 28:684-703.

15. Qiang Ma. Role of Nrf2 in Oxidative Stress and Toxicity. Annu Rev Pharmacol Toxicol. 2013; 53:401-26.

16. Fernández JL, Muriel L, Rivero MT, et al. The sperm chromatin dispersion test: a simple method for the determination of sperm DNA fragmentation. J Androl. 2003; 24:59-66.

17. Agarwal A, Saleh RA. Role of oxidants in male infertility: rationale, significance, and treatment. Urol Clin North Am. 2002; 29:817-27.

18. Gharagozloo P, Aitken RJ. The role of sperm oxidative stress in male infertility and the significance of oral antioxidant therapy. Hum Reprod. 2011; 26:1628-40.

19. World Health Organization. WHO laboratory manual for the examination and processing of human semen. 5th ed. Geneva: WHO Press; 2010.

20. Tournaye H. Evidence-based management of male subfertility. Curr Opin Obstet Gynecol. 2006; 18:253-9.

21. Tremellen K. Oxidative stress and male infertility-a clinical perspective. Hum Reprod Update. 2008; 14:243-58.

22. Showell MG1, Mackenzie-Proctor R, Brown J, et al. Antioxidants for male subfertility. Cochrane Database Syst Rev. 2014; 12: Art. No.: CD007411.CD007411.

23. Gorczyca W, Traganos F, Jesionowska H, et al. Presence of DNA strand breaks and increased sensitivity of DNA in situ to denaturation in abnormal human sperm cells: analogy to apoptosis of somatic cells. Exp Cell Res. 1993; 207:202-5.

24. Hughes CM, Lewis SE, McKelvey-Martin VJ, et al. Reproducibility of human sperm DNA measurements using the alkaline single cell gel electrophoresis assay. Mutat Res. 1997; 374:261-8. 
25. Evenson DP, Jost LK, Marshall D, et al. Utility of the sperm chromatin structure assay (SCSA) as a diagnostic and prognostic tool in human fertility clinic. Hum Reprod. 1999; 14:1039-49.

26. Agarwal A, Nallella K, Allamaneni S, et al. Role of antioxidants in treatment of male infertility: an overview of the literature. RBM Online. 2004; 8:616-27.

27. Zhou X, Liu F, Zhai S. Effect of L-carnitine and/or Lacetyl-carnitine in nutrition treatment for male infertility: a systematic review. Asia Pac J Clin Nutr. 2007; 16(Suppl 1):383-90.

28. Patel SR, Sigman M. Antioxidant therapy in male infertility. Urol Clin North Am. 2008; 35:319-30.

29. Ross C, Morriss A, Khairy M, et al. A systematic review of the effect of oral antioxidants on male infertility. RBM Online. 2010; 20:711-23.

30. Lafuente R, Gonzalez-Comadran M, Sola I, et al. Coenzyme
Q10 and male infertility: a meta-analysis. J Ass Reprod Gen. 2013; 30:1147-56.

31. Greco E, Iacobelli M, Rienzi L, et al. Reduction of the incidence of sperm DNA fragmentation by oral antioxidant treatment. J Androl. 2005; 26:349-53.

32. Martinez-Soto JC, Domingo JC, Cardobilla LP, et al. Effect of dietary DHA supplementation on sperm DNA integrity. Fertil Steril. 2010; 94:S235-236.

33. Menezo YJ, Hazout A, Panteix G, et al. Antioxidants to reduce sperm DNA fragmentation: an unexpected adverse effect. RBM Online. 2007; 14:418-21.

34. Bolle P, Evandri MG, Saso L. The controversial efficacy of vitamin E for human male infertility. Contraception. 2002; 65:313-5.

\section{Correspondence}

Luciano Negri, MD

luciano.negri@humanitas.it

Renzo Benaglia, MD

renzo.benaglia@humanitas.it

Emanuela Monti, MD

emanuela.monti@humanitas.it

Paolo E. Levi Setti, MD

paolo.levi_setti@humanitas.it

Humanitas Research Hospital, Department of Gynecology, Division of

Gynecology and Reproductive Medicine, Humanitas Fertility Center,

Rozzano-Milan, Italy

Emanuela Morenghi, MD

emanuela.morenghi@humanitas.it

Biostatistics Unit, Humanitas Research Hospital, Rozzano-Milan, Italy

Alessandro Pizzocaro, MD

alessandro.pizzocaro@humanitas.it

Endocrinology Unit, IRCCS, Humanitas Research Hospital,

Rozzano-Milan, Italy 